\title{
Structural and Organisational Conditions for Being a Machine
}

\author{
Guglielmo Militello • Álvaro Moreno
}

\begin{abstract}
Although the analogy between macroscopic machines and biological molecular devices plays an important role in the conceptual framework of both neo-mechanistic accounts and nanotechnology, it has recently been claimed that certain complex molecular devices (consisting of biological or synthetic macromolecular aggregates) cannot be considered machines since they are subject to physicochemical forces that are different from those of macroscopic machines. However, the structural and physicochemical conditions that allow both macroscopic machines and microscopic devices to work and perform new functions, through a combination of elemental functional parts, have not yet been examined. In order to fill this void, this paper has a threefold aim: first, to clarify the structural and organisational conditions of macroscopic machines and microscopic devices; second, to determine whether the machine-like analogy fits nanoscale devices; and third, to assess whether the machine-like analogy is appropriate for describing the behaviour of some biological macromolecules. Finally, the paper gives an account of 'machine' which, while acknowledging the physicochemical and organisational differences between man-made machines and biological microscopic devices, nevertheless identifies a common conceptual core that allows us to consider the latter 'machines'.
\end{abstract}

Keywords Machine-like systems · Macroscopic machines - Molecular machines • Mechanistic explanations $\cdot$ Nanotechnology $\cdot$ Biomolecular machines

\section{Introduction}

Over the last 25 years, the concept of 'machine-like system' has been extensively employed in the neo-mechanistic framework to describe biological mechanisms, since said mechanisms have been regarded as the functional components of a system which behaves like a machine. Moreover, the analogy between machines and certain biological macromolecular structures plays a key role in nanotechnology, with some kinds of macromolecules being artificially reproduced by considering them machine-like systems.

Neo-mechanistic accounts have so far focused on the epistemological aspect of mechanistic explanations in the life sciences, with a rough analogy often being drawn between (biological) mechanisms and machines. Nevertheless, as pointed out by Moore (2012), Skillings (2015) and Nicholson (2013), there are some relevant differences (mainly due to different size scales) in the physicochemical behaviour of macroscopic machines, on the one hand, and microscopic devices, on the other, that make this analogy rather dangerous. As a result, these authors have argued that the analogy between macroscopic machines and microscopic devices (such as synthetic nanomachines or certain biological macromolecules) should be taken with a grain of salt and, in most cases, completely dismissed. Yet the issue is far from simple, since the conceptual framework of contemporary nanotechnology is based on the idea that some biological macromolecules are indeed machines, and can therefore be artificially reproduced using a bottom-up approach, according to which a supramolecular structure may be built by assembling smaller molecular components. 
No comprehensive ontological analysis of the concept of machine and, particularly, the status of machine of certain kinds of microscopic devices (synthetic as well as biological) has yet been carried out by either neo-mechanistic accounts or the philosophy of (nano)technology. In an attempt to fill this void, this paper aims to establish the conceptual boundaries of the concept 'machine' and to understand to what extent some molecular devices may be defined as such. It is worth stressing that this paper is not aimed at claiming that organisms are machines, but rather at evaluating whether or not molecular synthetic devices and some biological macromolecular structures share common properties that make all of them 'machines'. In order to understand whether or some molecular devices are machines, it will be necessary to analyse the structural and physicochemical conditions of not only nanoscale devices, but also macroscopic machines, since the term 'machine' was originally coined to refer to macroscopic man-made devices (e.g. Archimedean simple machines), and only later, during the 20th century, was it applied to the domain of biological macromolecules.

In light of the above, the research questions to which this paper seeks to respond can be summarised as follows:

1. What are the structural and organisational features of artificial macroscopic machines, synthetic molecular machines and biological molecular machines?

2. To what degree does the 'machine-like' analogy fit a class of molecular devices operating at the nanoscale?

3. Is the 'machine-like' analogy appropriate for describing the operation of certain kinds of macromolecules in living cells?

An understanding of the ontological status of (nano)machines has two important explanatory consequences for the neo-mechanistic debate and nanotechnology. First, the clarification of the term 'machine' may shed some light on the biological mechanisms that are based on them ${ }^{1}$. Second, since the cornerstone of nanotechnology is the possibility of artificially reproducing certain biological macromolecules, the differences between biological and artificial molecular machines highlight the limits of its theoretical framework.

The structure of the paper is as follows. Section 2 presents and discusses neomechanistic accounts of 'machine-likeness'. Then, Section 3 analyses the features of artificial macroscopic machines. Section 4 offers a critical exploration of the structure and functioning of synthetic and biological molecular machines, and Section 5 focuses on the specific case of biological molecular devices, taking into account the criticisms and arguments put forward by Moore (2012), Skillings (2015), and Nicholson (2013) against the machine-likeness of nanoscale devices. Finally, Section 6 offers some concluding remarks.

\section{The concepts of machine and mechanism in neo-mechanistic accounts in biology. A critical review.}

\footnotetext{
${ }^{1}$ The term 'mechanism' is currently used in neo-mechanistic literature for designating both the (epistemological) problem of the explanatory power of mechanistic explanations (among others, Bechtel and Richardson (1993) 2010; Glennan 1996; Bechtel and Abrahamsen 2005) and the (ontological) organisation of -namely biological- mechanisms (among others, Machamer, Darden and Craver 2000; Craver 2001). When we state that we focus on the mechanisms "based on" (or performed by) machines, we merely claim that we describe, from an ontological perspective, the configuration of the mechanisms that are performed by the component parts of a specific kind of system (i.e. a machine). Hence, we do not address the issue of the explanatory power of mechanistic explanations.
} 
The idea that organisms can be explained through an analogy with machines is rooted in Descartes' thinking, as laid out in Discourse on the Method (1637 (1999)) and Treatise on Man (1664 (1972)). Since the publication of these seminal works, it has been widely assumed $^{2}$ that each anatomical part performs a distinct and specific biological function in the same (or at least, similar) way as the different parts of a machine make up a mechanism. The concepts of 'machine' and 'mechanism' are at the core of many biological descriptions (from genetics to evolutionary biology), and play a pivotal role in the neo-mechanistic view.

However, until recently, no precise definition of the term 'mechanism' had been developed. The first basic mechanistic account was clearly provided by Machamer, Darden and Craver $^{3}$ (2000), and has significantly influenced subsequent debates on not only the nature of biological mechanisms, but also the nature of machines. The MDC account defines biochemical mechanisms (e.g. neurotransmission and the mechanisms of DNA and RNA replication, transcription and translation) in terms of entities performing regular activities from start to finish conditions. Implicitly, this concept of mechanism is based on the way man-made machines work, since mechanisms have long been considered the functional parts of a machine-like system (Glennan 1996, pp. 5152; Bechtel and Richardson 2010, p. 17). Thus, as Nicholson points out (2012), one of the meanings sometimes carried by the concept of 'mechanism' is that of 'machine'.

Although these authors have developed a set of precise definitions for the concept of mechanism, they have not convincingly justified its relationship with the concept of machine. There are two main reasons for this. First, the development of a theory of machines has been essentially ignored by the advocates of mechanistic accounts in biology, who use the concept of mechanism in an epistemological-explanatory sense rather than an ontological one $e^{4}$. Consequently, the use of the machine analogy to explain biological systems has generally been supported by rather intuitive ideas about what a machine actually is. Second, some neo-mechanistic accounts have provided a very broad definition of 'mechanism' that encompasses both mechanisms which are based on machines and mechanisms which are not. Thus, the relationship between mechanisms and machines appears vague and unclear. We shall explain these two claims in more detail below.

In relation to the first aspect, the definitions of mechanisms offered by Bechtel and Richardson (2010) and Glennan (1996), while emphasising the fact that mechanisms behave like the functional components of a machine, fail to provide a detailed analysis and description of the ontological status of a machine, or indeed the machine-like behaviour of some biological macromolecules. Rather, they focus on the epistemological nature of mechanistic explanations and, collaterally at least, the epistemological aspect of machine-likeness (i.e. the fact that a machine may be explained through mechanistic accounts). In the same vein, Levy (2014) links the concept of 'machine-likeness' to decompositional strategies 5 , since a machine can be decomposed by virtue of two features: first, the differentiation of parts (Levy 2014, p. 5); and second, the local relations among the component parts (Levy 2014, pp. 5-6). In other words, modularity and internal interactions among the local functions of a system

\footnotetext{
${ }^{2}$ This is not to say that this view has not met with strong opposition (i.e. vitalism and, later, organicism).

${ }^{3}$ Hereinafter, we shall refer to Machamer, Darden and Craver's account as the MDC definition.

${ }^{4}$ Although Illari (2013) stresses that Bechtel's view is epistemic whereas Craver's account is ontic, we will not address this issue here. Instead, we will examine why a number of (mainly epistemological) accounts of (biological) mechanisms have not so far focused on the ontology of (nano)machines.

${ }^{5}$ By decompositional strategies we mean an epistemological account of the behaviour of a system in terms of the local behaviour of its subsystems (component parts) and their causal interrelations (compare Bechtel and Richardson, 1993 (2010).
} 
provide it with a certain degree of order, as well as decomposability, which in turn allow it to be defined as a 'machine'. In spite of their importance, however, these aspects do not shed any light on the ontology of a machine.

As regards the second claim, the MDC definition of mechanism in terms of 'entities and activities organised such that they are productive of regular changes from start to termination conditions' (MDC 2000, p. 3) is much broader and encompassing than the conceptual core of the operation of a machine. Here again there are two main reasons for this. First, because the component parts of a machine (the 'entities') are not only organised, but also held together in a (meta)stable structure, nearly in thermodynamic equilibrium ${ }^{6}$. Second, because 'the activities' of the components of a machine take place only when an input of energy occurs and are aimed at displacing a force, doing work or performing a function. Accordingly, the mechanism of a machine needs a thermodynamically-stable structure, and this requirement is not included in the MDC definition. An MDC mechanism could be either the result of the activities of parts organised in a thermodynamically-stable structure (and would therefore coincide with our concept of the mechanism of a machine), or the result of a far-from-equilibrium organised set of coupled processes. Many biochemical pathways indeed produce a functional activity (that which the MDC account defines as 'mechanism'), which may be explained as resulting from clearly distinguished 'parts' (i.e. the chain of reactions catalysed by specific enzymes), understood as processes. However, as shown in the following sections, this kind of mechanism is not compatible with our concept of the mechanism of a machine, because a biochemical pathway fails to exhibit some important features of machines, such as a thermodynamically-stable structure or an energy input to do work. For these reasons, the basic mechanistic account provided by MDC does not clarify the difference between those mechanisms which are based on machines and those which are not.

Usually neo-mechanistic accounts (notably Bechtel and Richardson (1993) 2010; MDC 2000; Craver 2001; Bechtel and Abrahamsen 2005) use the term 'organisation' to refer to the specific way the different parts of a machine are arranged so as to perform a given function. The use of this term is however a bit ambiguous. For, on the one hand, strictly speaking, the component parts and the operations of a machine may be said to be (structurally, spatially, and temporally) ordered. Yet, on the other hand, in order to perform a function, they should contribute to the maintenance of a system to which they belong (i.e., they are generated in this system, and they contribute to its maintenance). More precisely, we say that a machine performs a function insofar as it is embedded in a context (for example a specific social organisation) where certain material structures (i.e. machines) are produced. If machines are rightly designed and fabricated, they can also contribute to the maintenance of the context itself (for example the life of society to some extent depends on the existence of machines). And in a similar vein, certain macromolecular devices in the cell perform a function because they are embedded in the cellular 'organisation', which they contribute to maintain and where they are produced. It is a human 'organisation' that produces an artificial machine and provides them with a specific function; and it is a biological 'organisation' that produces a molecular machine and provides them with a specific function. In both cases the term 'organisation' is what justifies that a given composite material structure, constraining a

\footnotetext{
${ }^{6}$ By this we mean that the structure of a machine (i.e. the specific assemblage of its component parts) would be preserved even if the exchange of matter and energy with its surroundings were almost zero (i.e. thermodynamic equilibrium). The stability of a structure is different from the functionality of a machine, because functionality requires an exchange of matter and energy between the machine and its surroundings.
} 
flow of energy, achieves a function (see for details Mossio et al 2009 and Moreno and Mossio 2015). Derivatively, it would be sensible to say that the ordered structure of the functional parts constituting a machine is also "organised" in order to fulfil the global function performed by the machine as a whole.

In sum, the (neo)mechanistic use of the term 'mechanistic explanation' is much more liberal than ours, as we focus only on the mechanisms performed by machines. Since the purpose of this paper is to conduct an ontological examination of the concept of 'machine', we will not enter here into current (and important) debates about the explanatory validity or limits of the (neo)mechanistic accounts, particularly in light of the challenges raised by the success of network-like explanations, which are usually incompatible with the idea of functional decomposition (Zednik 2011; Kaplan 2015; Bechtel 2017).

Although the neo-mechanistic debate has so far devoted most of its attention to the epistemology of biological mechanisms, there is still a long tradition of studies on the structure and functioning of man-made machines. Serious attempts to define what a machine actually is can be traced back to the second half of the 19st century, when the German engineer Franz Reuleaux developed a theory which posited that a machine is a kinematic chain of elementary links called 'kinematic pairs'. In his book 'The Kinematics of Machinery', the term 'machine' refers to a system that converts an energy input into an energy output by exploiting the mechanism(s) of its component parts that displace an applied force and, therefore, do work (Reuleaux 1876) ${ }^{7}$. The functional components of a machine exhibit a specific design that allows them to harness the physicochemical processes underlying the behaviour of said machine. In other words, a machine is a set of functional constraints that are interlocked so as to harness the action of physical laws in order to achieve a new (composite-integrated) function, as pointed out by Polanyi (1968). To do so, a functional hierarchy must be established, and a spatial and temporal order must be imposed on the functional constraints. As Wimsatt (1986) highlighted, the functional components of many machines can be partially intersubstituted within a certain range of configurations and without changing systemic properties. As a result, the nature of a composite-integrated function of a machine is determined by the structure and functions of its constituents (principle of compositionality). A crucial feature of machines is that they consist of a number of modular parts that are assembled according to a specific design so as to assume a distinctive shape. Accordingly, the pieces of a machine can be isolated due to modularity, and are gathered in a very specific way in order to perform a certain function. Another essential feature of a machine is its compactness, namely the structural co-dependence of its component parts, which is a result of the design of the machine. Compactness allows a machine to exhibit clear boundaries that distinctly distinguish it from its surroundings.

In the light of the above, we recognise that there is a tension between the concepts of 'machine' and 'mechanism' in the current neo-mechanistic framework. We propose to resolve this ambiguity as follows: we define a machine as a meta-stable structure, which can persist in thermodynamic equilibrium, consisting of a number of functional interdependent parts that constrain an energy flow to do work and perform a systemic function. We characterise a mechanism performed by a machine as the set of all functions carried out by the component parts of the machine that allow it to harness a flow of energy and matter and to do work. In other words, 'machine' designates a

\footnotetext{
${ }^{7}$ This author defined a machine as 'a combination of resistant bodies so arranged that by their means the mechanical forces of nature can be compelled to do work accompanied by certain determinate motion.' (Reuleaux 1876).
} 
certain kind of a structure, whereas a 'mechanism based on a machine' refers to its systemic functionality. The mechanism of a machine is the result not only of the specific structure of a machine, but also of a human or biological context that provides a machine with a specific (structural) order of its component parts and a particular mechanism. Indeed, to a certain degree, one can abstract the functioning of a machine from its material and organisational embodiment. Yet, although features such as design, structural stability, shape, compactness, modularity and compositionality pertain to the structure (i.e. to machine), but not its functionality (i.e. its mechanism), they should indirectly inform our understanding of a mechanism also. As a matter of fact, the mechanisms of each machine constrain a flow of energy by virtue of the specific shape of the component parts of a machine and the way in which they are ordered.

For these reasons, in this paper we will focus on the nature of machines (what they are and what aspects define their operations) and analyse to what extent the machineanalogy can be applied to the core of all living organisations, i.e. the cell. It is true that biological machines are microscopic and their physicochemical properties are very different from those of macroscopic machines. But before analysing the implications of the nanoscale, we shall first clarify what a machine is by analysing the example of artificial macroscopic machines.

\section{Artificial macroscopic machines ${ }^{8}$}

The oldest and simplest macroscopic machines can be traced back to Archimedean simple machines (e.g. levers, screws and pulleys, etc.), which are devices that modify the direction or magnitude of a force in order to do work against a single load force. Simple machines are often considered the building blocks of more complex "compound machines'. Power sources are exploited to transmit power' or transform motion and, therefore, perform a mechanism ${ }^{10}$. Both simple and compound machines do work by harnessing a flow of energy into an ordered process so as to achieve a pre-specified function $^{11}$. This is made possible by a set of specific material structures, which act as constraints, functionally harnessing the flow of energy so as to produce a forward motion. When a macroscopic machine is at work, the summation of all external forces and torque is not zero (the machine is far from mechanical equilibrium). Since the movement and the work of a macroscopic machine are the outcome of the relative internal motion of its component parts, they must be assembled in an ordered way (following specific design rules) in order to achieve a functionally-integrated operation. This is commonly referred to as the 'structure' of a machine.

The design of a macroscopic machine is closely linked to its functionality, insofar as shape, form, and size scale determine certain kinds of mechanisms and not others. According to Reuleaux (1876), a machine consists of an assemblage of resistant bodies (links), which are connected together (the so-called 'kinematic pairs') by movable joints

\footnotetext{
${ }^{8}$ In this section we mainly refer to mechanical machines such as steam machines, cars, pumps, etc. Of course, there are many other kinds of non-mechanical machines (e.g. computers) which we have not described here, because all of them share the same basic features of what we have called 'machine'. Hence, for the sake of simplicity, we focus on mechanical machines as paradigmatic examples of artificial macroscopic machines.

${ }^{9}$ Power is the transmission of energy from the place where it is generated to another place, so as to perform useful work.

${ }^{10}$ In machine theory, when one link is chosen as the framework of reference for the movement of all other links, it is called the 'frame link'. Once a frame link is set out in a kinetic chain, and it is possible to generate an output motion in response to an input motion, the kinetic chain is called a 'mechanism'.

${ }^{11}$ Needless to say, the function of an artificial macroscopic machine is specified by its designer.
} 
so as to form a kinematic chain with one link fixed and having the purpose of transforming motion. Reuleaux's characterization of machines primarily encompasses mechanical devices and, therefore, considers component parts as rigid structures. However, many contemporary machines exhibit constituents which are not rigid, but rather flexible, such as magnetic parts (e.g. in an electromagnetic coil), fluidic components (e.g. in a refrigerator), and so forth. The links of a machine are structures that move in the air or in a vacuum by exhibiting relative motion that is constrained by the number of links, the type of joint used to connect them and the shape of the mating surfaces. Each link is connected to the other links through joints that transmit movement from the input link ('driver') to the output link ('follower'). Since each link is aimed at maintaining constant spatial relationships between the elements of its pairs (Dicker et al. 2003 , p. 6), the way in which the pieces of a machine are assembled together is crucial to defining the mechanism, the work, and the kind of function performed. As a matter of fact, the overall function of a machine hinges on the compositionality of the local functions performed by its parts. A good example of a macroscopic machine design is a gear pump, which exploits the rotation of gears to displace fluids. A gear pump consists of two gears (links) that are connected through a contact zone (movable joint) which allows two gears to pivot with respect to each other in such a way that they form a kinematic chain. In order to work properly, each gear must maintain a specific angle with respect to the other one (constant spatial relationship). It is important to underline that a key requirement for macroscopic machines is that the parts be structurally codependent, so that the overall organisation is stable and, at the same time, compact, with clear spatial boundaries.

The structure of a macroscopic machine (i.e. the structural interdependence among its parts) may be said 'stable', because it is maintained regardless of whether or not the device is actually doing work (and performing a function). For example, the structure of a refrigerator or a car is stable, since it is maintained regardless of whether or not these machines are switched on or off (i.e. if they actually work or not). Then, macroscopic machines may be defined as 'compact', because they exhibit a specific design and their component parts are assembled in such a way to be closely and firmly united in a distinct pattern. For example, the component parts of a refrigerator or a car are closely interlocked in such a way that they have a compact aspect. Finally, the component parts of a macroscopic machine show clear spatial boundaries, because their different pieces are assembled in a specific way so as to build a macroscopic device. For instance, a refrigerator is composed of clear distinct assembled parts such as a thermally insulated compartment and a heat pump that transfers the heat from the inside to the outside of the refrigerator.

Thus, the component parts of an artificial macroscopic machine perform a mechanism because of the ordered structure of their constraints. This ordered structure of constraints is evident in the way in which the links are assembled (design) so as to channel the motion of each part in a certain direction. The structure of constraints is designed so as to minimise the inertial and friction forces acting on the parts (i.e. the links) of the machine. Friction forces, which act on the mating surfaces between two links, affect the motion of the parts of a macroscopic machine, because friction forces (i.e. dry friction) determine the tractive force between a body and a tangential force. Much the same occurs with inertial forces, which oppose any change in the velocity of motion or the torque of a rigid body. Since friction and inertia influence the sliding velocity of the mating surfaces of the links and any changes in their velocity (respectively), the overall movement, and thus the mechanism, of a macroscopic machine is inevitably affected by these physical forces. 
All in all, to constitute a mechanism in a macroscopic machine, each link of a kinematic chain must exhibit a specific shape and dimension, as well as a distinct connection with the other links in such a way as to ensure a certain degree of freedom $(\mathrm{DOF})^{12}$ and, therefore, perform a relative motion. Since design is crucial to enabling the component parts to work and to perform a certain function, the links of a machine (e.g. wheels, gears, cams and pistons, etc.) must be assembled in a particular way so as to perform a certain kind of mechanism and a specific function. For example, a four-bar linkage (see Fig 1 A four-bar linkage: $\mathrm{O}_{2}, \mathrm{~A}, \mathrm{~B}$, and $\mathrm{O}_{4}$ are the joints that allow links (2, $3,4)$ to move with a specific angle $(\beta$ and $\gamma)$. The link 2 is the input link and the link 4 the output link. (Simón 2016, p. 15)) is a mechanism that can perform a wide variety of movements depending on how the four links are assembled and connected together: it can be employed in a pumpjack to draw oil from the subsoil by using a planar quadrilateral linkage; or alternatively, it can be used in a train suspension mechanism to allow the wheel to rotate through a slider-crank linkage. In short, the concepts 'mechanism', 'function' and 'work' in a macroscopic machine should be understood in terms of how the component parts are assembled so as to achieve a functionallyintegrated action.

\section{[Insert here Fig 1]}

\section{Molecular machines}

Having clarified the core concept of 'machine', we will now turn to what are often referred to as 'molecular machines'. Here we find two very different systems: 'molecular machines' and biological 'molecular machines', which while sharing many features, also diverge in many other important ways. For this reason, we shall divide our analysis into two parts. Firstly, we shall argue why, despite the specific differences generated by the nanoscale, it is still correct to talk about machines at the molecular scale. And secondly, and perhaps more importantly, we shall explore why it also makes sense to classify certain macromolecular structures operating in cells as machines.

Let us begin by considering, from a generic perspective, the current view regarding what a 'molecular machine' (MM) actually is. First of all, an MM is defined as any discrete number of molecular components that produce quasi-mechanical movements (output) in response to specific stimuli (input) (Ballardini et al. 2001). Unlike macroscopic machines, the configuration space of MMs is not defined by their six degrees of freedom ${ }^{13}$, but rather by their free-energy landscapes ${ }^{14}$ (i.e. Gibbs free energy ${ }^{15}$ of interacting molecules) (Astumian and Hänggi 2002; Astumian et al. 2016).

More specifically, MMs are characterised by three important elements: firstly, thermal noise; secondly, structural anisotropy; and, thirdly, an energy input (Astumian 2002). Thermal noise ${ }^{16}$ acts as 'thermal activator' of MMs, since it provides them with an

\footnotetext{
12 The degree of freedom (DOF) of a mechanical system is defined as 'the number of independent parameters that unambiguously define its position in space at every instant' (Simón et al. 2016, p. 2).

${ }^{13}$ The six degrees of freedom of a macroscopic rigid body are defined by three rotatory movements (roll, pitch, yaw) and three translational movements (surge, heave, sway).

${ }^{14}$ The energy landscape is the mapping of all possible spatial conformations of a molecule. The energy landscape is a continuous function that associates each physical state of the molecule with the corresponding energy.

${ }^{15}$ Gibbs free energy is a thermodynamic potential used by a thermodynamic system to do work at a constant temperature and pressure. The simple equation for Gibbs free energy is: $\Delta \mathrm{G}=\Delta \mathrm{H}-\mathrm{T} \Delta \mathrm{S}$, where $\Delta \mathrm{H}$ is the enthalpy change and $\Delta \mathrm{S}$ is the change in entropy of the process.

${ }^{16}$ Thermal noise is the electronic noise determined by the thermal agitation of the charge carriers.
} 
amount of energy to overcome energy barriers ${ }^{17}$. If the noise intensity is low, molecules are pinned at a potential minimum and they cannot diffuse; on the contrary, if the noise intensity is high, molecules overcome the potential barrier and begin to diffuse (Astumian 2002; Astumian and Hänggi 2002). Thermal noise randomly 'pushes' an MM back and forth without a specific direction. Nevertheless, MMs exhibit a directional movement by combining structural anisotropy with an energy input (Astumian and Hänggi 2002). Structural anisotropy is the asymmetric distribution of reaction products around an $\mathrm{MM}$ and it acts as an asymmetric kinetic barrier. When an energy input (chemical, photochemical, etc.) is provided, structural anisotropy generates a concentration gradient of chemical potential that constrains Brownian motion and generates a directed motion of an MM. Thus, as a result of the interplay between thermal noise, structural anisotropy, and an energy input, an MM is able to functionally harness an energy source, constrain Brownian motion and perform a (biological) task ${ }^{18}$.

It is crucial to emphasise that MMs usually operate in aqueous solutions where they are subject not only to important thermal fluctuations, but also to viscous forces that render inertial ones negligible. Since the role played by viscous forces is completely different at the macroscopic and microscopic levels, the Reynolds number ${ }^{19}$ (i.e. a dimensionless parameter comparing the effect of inertial and viscous forces) is different for macroscopic and microscopic devices. Macroscopic machines have a high Reynolds number, and inertial forces are important whereas viscous ones are negligible. Microscopic machines, on the other hand, have a low Reynolds number, meaning that viscous forces are fundamental and inertial forces negligible within the system.

Unlike macroscopic machines, MMs operate very near to mechanical equilibrium because the viscous drag force ${ }^{20}$ is equal and opposite the net mechanical force. The 'mechanical equilibrium' of a molecular system is a dynamic condition in which every forward motion of a particle is cancelled by its microscopic reverse (i.e. a backward motion) (Astumian 2012), and it is therefore different from the concept of thermodynamic equilibrium. Accordingly, the presence of a ratchet mechanism in an MM allows it not only to direct movement but also to keep the system very near to, but not at, mechanical equilibrium. Although MMs are close to mechanical equilibrium, they are far from thermodynamic equilibrium, since they dissipate energy to their environment.

Unlike macroscopic machines, which exploit many different energy sources (mechanical, thermal, chemical, electrical, etc.), MMs consume chemical, photochemical, and electrochemical energy. Chemically-driven MMs are subjected not only to thermal noise but also to the principle of microscopic reversibility, according to which at equilibrium the forward and backward paths of a reversible reaction are equally likely to occur. In order to overcome microscopic reversibility, chemicallydriven MMs cyclically switch between different mechanical states, a process known as 'chemical gating', during which the selective binding/unbinding of a catalyst allows the device to increase its chemical potential and modify the reaction rate constant in such a

\footnotetext{
${ }^{17}$ Energy barrier (or activation energy) is the least amount of energy required to trigger a chemical reaction.

${ }^{18}$ Feynman (1963) pointed out that it is impossible to have a molecular device (the so-called 'Brownian ratchet') that is able to extract work from thermal noise because of the inviolability of the second law of thermodynamics. However, a molecular machine does combine thermal noise with structural anisotropy and energy (chemical, photochemical, and electrochemical) sources to do work. For this reason, MMs are also called 'Brownian ratchets' (Astumian 2002).

${ }^{19}$ Reynolds number is expressed by the ratio between $a v \rho$ and $\eta(R=a v \rho / \eta)$; where $a$ is the acceleration, $v$ the velocity, $\rho$ the density of the fluid, and $\eta$ the fluid's viscosity (Astumian and Hänggi 2002, p. 33).

${ }^{20}$ Viscous drag force is the force exerted by a fluid on an obstacle around which it flows.
} 
way that the reaction can follow only one path (forward or backward). As a result, the mechanochemical cycle of binding/unbinding a catalyst is the way in which chemicallydriven MMs constrain a chemical energy input in order to carry out directional movement, do work, and bypass microscopic reversibility (Astumian 2012; Astumian et al. 2016).

Unlike chemically-driven MMs, light-driven ones exploit the allosteric conformational change generated by exergonic reactions (known as 'power stroke') to allow light energy to maintain a non-equilibrium steady state, thereby permitting molecules to move between two separate energy surfaces (Astumian et al. 2016). Another significant difference between chemically and light-driven machines is microscopic reversibility, since the former are subject to microscopic reversibility whereas the latter are not (Astumian et al. 2016).

In light of all these factors, it is sensible to avoid a hasty analogy between MMs and all types of macroscopic machines. A careful analysis is therefore required to assess the question. In the following two subsections we will analyse the structural and physicochemical organisation of both artificial (Section 4.1) and biological (Section 4.2) MMs.

\subsection{Artificial MMs}

Since the beginning of the 21st century, a host of molecular devices have been artificially developed for technological use in different domains (nano-medicine, green nanotechnology, etc.) and with very different purposes. Nanotechnology can be considered an extension of supramolecular chemistry, a new avenue opened up during the 1970s (Lehn 1995). Artificial MMs (also called 'supramolecular structures') are built by assembling a discrete number of molecular components with the aim of performing a function through the mechanical movement of their parts. Energy sources are provided by photochemical and electrochemical energy inputs that cause exergonic reactions ${ }^{21}$, which in turn power these artificial nano-devices. Photochemical and electrochemical energy is transformed into mechanical work through a 'motor-like' part.

Unlike macroscopic machines, MMs are built by harnessing the intrinsic selfassembly capacities of certain molecular components, according to which these components bind together through non-covalent interactions in such a way that the final assembled structure is able to perform mechanical movements (linear, rotatory, oscillatory, etc.), thus enabling a specific function to be carried out. This method for building an MM is called bottom-up assembly ${ }^{22}$. Artificial nano-machines are based on rotaxanes, catenanes and other related structures (Sauvage and Dietrich-Buchecker 1999; Balzani et al. 2005) which are assembled by employing non-covalent interactions such as hydrogen bonding, coulombic forces and metal-ligand bonding, among others. Rotaxanes are dumbbell-shaped molecules surrounded by a macrocyclic compound with a ball at each end; catenanes consist of two interlocked rings (macrocycles) (Balzani et al. 2005) (see Fig 2 Interactions between a rotaxane and a macrocycle: a) ring shuttling, b) ring rotation, c) threading/dethreading equilibrium between a macrocycle and the axle of a pseudorotaxane (Credi et al. 2014, p. 6)).

\section{[Insert here Fig 2]}

\footnotetext{
${ }^{21}$ Endergonic reactions can also occur, but they have to be thermodynamically coupled with exergonic reactions in such a way that exergonic reactions drive or power endergonic ones.

${ }^{22}$ By a 'bottom-up' approach to molecular machines, we mean the construction of nanoscale devices and machines using a molecule-by-molecule method (Balzani et al. 2005).
} 
Like macroscopic machines, synthetic nano-devices carry out work and perform a function by virtue of the way certain molecular parts have been shaped and located so as to affect the relative motion of other component parts and, all together, harness the energy flow in a specific way. It is the interlocked architecture of the components (i.e. their design and structural co-dependence) that permits the overall system to transform an energy input into work, in order to perform a desired function. Like the links of a macroscopic machine, rotaxanes and catenanes generate relative motion ${ }^{23}$ as the result of an energy input. Both mechanical movements and a variety of different functions of the molecular components of rotaxanes and catenanes are induced by external stimulation. For example, acid-base chemical inputs may strengthen or weaken the hydrogen bonding interactions that are responsible for assembly and spatial organisation. Another important physical constraint on the behaviour of rotaxanes and catenanes is represented by non-covalent interactions, since these interactions allow them to bind to one another reversibly. Since non-covalent interactions easily bind (and unbind) the component parts of a synthetic nano-device, supramolecular stability hinges on the control of these weak interactions. Thus, the basic principle underlying the construction of artificial MMs is control of the non-covalent interactions that govern the relative mechanical movement of the building blocks so as to create a functionallyintegrated structure that is able to perform work, transport cargoes or signal molecules through molecular shuttles, etc. (Valero et al. 2017).

One example of artificial MMs is DNA nanotechnology (see Fig 3 Representation of a DNA architecture a) double strand DNA rotaxane with spherical stoppers; b) controlled release of the rings; c) a gold ( $\mathrm{Au}$ ) nanoparticle hybridizes two DNA rotaxanes; d) DNA origami rotaxane (Valero et al. 2017, p. 161)), which combines rotaxanes, catenanes and related structures to create interlocked DNA structures that can be generated from both double-stranded and single-stranded DNA (Ackermann et al. 2010; Valero et al. 2017).

\section{[Insert here Fig 3]}

\subsection{Biological MMs}

Biological MMs are a subgroup of macromolecules (mainly proteins) that are commonly found in both prokaryotic and eukaryotic cells. Noteworthy examples include molecular motors (such as dynein, myosin and kinesin), molecular pumps (such as transmembrane ATPases), molecular tweezers (such as DNA) and molecular switches (like rhodopsin) ${ }^{24}$. In the cellular environment, proteins are the molecular structures best suited to acting like 'machines', because their structure allows them to perform a wide variety of biochemical functions (from catalysis to cell signalling and signal transduction, and from cellular motility to ligand binding).

Here, we will analyse only biomolecular motors and pumps, since they are the best candidates to be considered MMs. There are two crucial features of biomolecular motors and pumps to take into consideration. First, like synthetic nano-devices, biomolecular motors and pumps emerge from self-assembly processes by harnessing the entropic effect generated by the translational displacement of the water molecules in the cytoplasm. Self-assembly occurs spontaneously if Gibbs free energy is negative

\footnotetext{
${ }^{23}$ Rotaxanes and catenanes usually perform relative motion through the movements of rings, such as shuttling along the axle of the rotaxane dumbbell or rotation around another ring in a catenane structure.

${ }^{24}$ Whereas molecular motors are able to displace unidirectionally when powered by an external energy input, molecular tweezers hold items between their two arms. A molecular switch reversibly shifts between two or more stable states.
} 
(O'Mahoni et al. 2011). Since an increase in the entropy of the water molecules decreases their Gibbs free energy, the self-assembly process is stimulated within the cytoplasmic environment (Kinoshita 2016). And, second, since modularity lies in the fact that biomolecular motors and pumps are proteins, and proteins consist of modular parts (Trifonov and Frenkel 2009; Rorick and Wagner 2011), another important feature of most of MMs is modularity. As a matter of fact, they consist of a number of subunits, each with a specific size and form, which are integrated in order to keep the global structure stable and transform chemical energy into mechanical work by means of a mechanochemical cycle. Three examples of biomolecular motors are myosin, kinesin and dynein, on the one hand, and an example of pump is the $\mathrm{F}_{0} \mathrm{~F}_{1}$ ATPase, on the other.

Kinesin, myosin and dynein ${ }^{25}$ are polymers generated by the self-assembly of their respective monomers. The movement of these biomolecular motors is due to a series of mechanochemical cycles during which a phosphoryl group, removed by ATP hydrolysis, causes a rearrangement of the elements of the ATP-binding site in the globular head, which in turn triggers structural changes in the track binding site. Next, the electrochemical energy generated by the motor domain is transduced by the neck domain into mechanical work by producing movement. When a phosphoryl group is released, a conformational change occurs in the globular head and the mechanochemical cycle ends.

$\mathrm{F}_{0} \mathrm{~F}_{1}$ ATPase (see Fig 4 Regions, subunits, and rotatory movement of $\mathrm{F}_{0} \mathrm{~F}_{1}$ ATPase: $\mathrm{F}_{0}$ region (subunits a and c), $F_{1}$ region (the other subunits) (Wilkens 2000, p. 338)) is a protein located in the inner mitochondrial membrane, which is synthesised by assembling a number of monomers into eight subunits and two functional regions $\left(\mathrm{F}_{0}\right.$ and $F_{1}$ ). Since the function of regions $F_{0}$ and $F_{1}$ is likened to that of the stator and rotor (respectively) of an electric motor, $\mathrm{F}_{0} \mathrm{~F}_{1} \mathrm{ATPase}$ is considered a vivid illustration of a biomolecular motor. The $\mathrm{F}_{0}$ subunits channel a proton flux, determined by an electrochemical gradient, which is exploited to allow $F_{1}$ to rotate. The rotatory movement is not random (but rather directed by subunits a and $c$ of $F_{0}$ ) and determines the conformational change of subunit $\beta$ of $F_{1}$, thus enabling the synthesis of ATP molecules (see Fig 5 Conformational changes of the subunit $\beta$ of F1 in order to synthesize ATP molecules (Feniouk and Yoshida 2008, p. 283)).

\section{[Insert here Figg 4 and 5]}

A biological MM exists and performs work not only because of self-assembly and modularity, but also due to three structural principles. First, reactions occurring in the different subunits are sequentially ordered so as to form a clear-cut biochemical pathway. Second, macromolecular conformational change, which is allosterically regulated, is temporally coordinated with the reactions occurring in the other subunits of the protein complex. And finally, and this is the most important point, the overall function of a biomolecular machine depends on its relationship with other biological molecules that are present in the biochemical network of the cell. These structural principles underlie the behaviour of all biomolecular machines. By way of example, let us again consider $\mathrm{F}_{0} \mathrm{~F}_{1} \mathrm{ATPase}$. The rotation of the $\gamma$ subunit of the $\mathrm{F}_{1}$ region may occur only if the subunits of the $\mathrm{F}_{0}$ region have previously constrained the proton flux towards the $\mathrm{F}_{1}$ region (sequential order). In order to produce three ATP molecules, the rotatory movement of $F_{1}$ must be coupled with the conformational change (three states) of

\footnotetext{
${ }^{25}$ Dynein is a protein that transports cargoes along microtubules in a cell by exploiting retrograde transport. Myosin is a protein that allows muscle contraction by interacting with actin. Kinesin is a protein that transports cargoes by sliding down microtubule filaments (anterograde transport).
} 
subunit $\beta$, in such a way that the phosphorylation of ADP generates an ATP molecule (temporal coordination). It is important to stress that the rotation of $\mathrm{F}_{1}$ must be coupled with ATP hydrolysis, otherwise, a futile cycle occurs without ATP production. Finally, since the electrochemical gradient proton flux through the ATP synthase depends on the electron flux produced by the electron transport chain, the overall function of $\mathrm{F}_{0} \mathrm{~F}_{1} \mathrm{ATPase}$ hinges on the biochemical pathways established in the protein complexes of the electron transport chain (relationship with other biological molecules).

The interdependence between a biological MM and the cell network is a key aspect that distinguishes MMs from artificial nano-devices. The functional integration of a biomolecular machine into the cellular network is a crucial organisational feature that makes it difficult to separate a biomolecular machine from its biochemical network, while at the same time explaining why artificial molecular machines are still a long way from being similar to biological ones. Biological MMs are embedded in a biochemical network in such a way that they appear functionally integrated into other biomolecular machines or biological macromolecules. This third characteristic is a key difference between artificial nano-devices and biomolecular machines, because synthetic nanomachines have not so far been incorporated into artificial biochemical networks. Consequently, whereas the energy input of biomolecular motors is constantly provided by the biochemical network in such a way that biological machines regenerate, synthetic nano-machines cannot do this, and therefore need an opposite input to reset (Balzani et al. 2005; Credi et al. 2014).

\section{Machine-likeness at the nanoscale}

In the previous sections, our analysis of the structural and physicochemical conditions required by macroscopic and molecular machines has revealed that both types share a fundamental similarity in their organisation, since both are meta-stable structures consisting of functional parts that constrain an energy input so as to perform work and, therefore, fulfil a systemic function. This similarity is the main reason why a machinebased terminology is so widely used in the specialist literature to characterise these types of artificial and biological molecular systems.

Admittedly, this is not a sufficient reason to dismiss the importance of the differences which exist between classic macroscopic machines and their molecular analogues. As a matter of fact, several critical voices have recently raised fundamental objections to the consideration of molecular devices as machines. To be fair, however, these criticisms are directed mainly at biological molecular machines, and fail to address (explicitly, at least) the case of their artificial counterparts. Yet, since many of these criticisms discuss aspects linked purely to scale differences, we believe they implicitly include a rejection of the adequacy of a machine-based terminology to describe artificial molecular devices also. In this section we will discuss the criticisms levelled by three authors: Moore, Skillings and Nicholson, before presenting our own view of the question. Whereas the arguments espoused by the first two authors focus exclusively on scale differences (and therefore, even though they only explicitly discuss the case of biological MMs, their arguments encompass artificial MMs also), Nicholson's criticism raises questions which pertain only to biological MMs. Thus, in our own analysis, we shall attempt to distinguish which part of the discussion specifically concerns only the biological case.

In a paper published in 2012, Peter Moore argues that macromolecules cannot be considered molecular machines because they are subject to physicochemical forces that are different from those of macroscopic machines, a circumstance which makes the analogy between macroscopic machines and macromolecules inappropriate. Moreover, 
he adds that "the use of the word "machine" is pernicious because of its implication that the functional properties of macromolecules can be explained mechanically, which is simply not true' (Moore 2012, pp. 7-8). Moore is certainly right in claiming that the physicochemical laws underlying macroscopic machines are different from those of microscopic macromolecules, because a different size scale entails a great difference both in the structure and in the functions performed by these two kinds of device. As seen in Section 4, the behaviour of both biological and synthetic macromolecular devices is influenced by viscous forces, thermal noise and potential energy differences in the free-energy landscape of macromolecules, etc. Together, these factors make the behaviour of these macromolecular structures probabilistic, not deterministic, because the laws of quantum mechanics replace Newton's laws of mechanics. In this sense, Moore is right in saying that the expression 'Brownian ratchet' should not be read in the deterministic sense of Newtonian mechanics (Moore 2012, p. 10), but rather as a linguistic label to simplify the interplay between structural anisotropy and an energy input to harness thermal noise. The criticism levelled by Moore (2012, p. 7) at 'structure-based movies' ${ }^{26}$ of macromolecules is also fair, insofar as they are indeed an oversimplification of how real macromolecules (e.g. ribosomes, myosin, dynein, $\mathrm{F}_{0} \mathrm{~F}_{1} \mathrm{ATPase}$ and so forth) generate motion and carry out work. In other words, Moore is right in claiming that the directional movement of macromolecules is not the same as that of a macroscopic machine (a car, for instance), because motion at the nanoscale is stochastic, not deterministic.

However, we do not agree with Moore's argument that these differences preclude the possibility of talking about (certain types of) macromolecular systems in terms of machines. Although they are indeed different from macroscopic machines due to the action of diverse physicochemical forces, they nevertheless share a common organisation. As we have seen, both macroscopic machines and the microscopic (biological as well as synthetic) devices studied so far are characterised by a number of functionally-ordered component parts that act as constraints on an energy input in order to do (useful) work. Moore (2012, p. 9) maintains that the operation of the component parts of a macromolecular 'machine' (e.g. the two subunits of a ribosome) are not directly related to their function because thermal fluctuations 'separate one functionally significant event from the next' (Moore 2012, p. 9). Thermal noise indeed distinguishes between macroscopic and microscopic causal sequence (which is deterministic in the former and probabilistic in the latter), but this does not prevent the global result of the device from being explained in terms of a specific sequence of functional operations. Hence, the specific way in which a macromolecular device behaves (e.g. the ribosome function of synthesising peptides) is due to the sequential organisation of a number of functions that are locally performed by the component parts of that same macromolecular device (e.g. the two subunits of a ribosome). Like macroscopic machines, microscopic ones carry out systemic functions by virtue of the organisation of the local functions fulfilled by their component parts.

The aim of Skillings' (2015) paper is to show the limits of the basic mechanistic account in explaining molecular processes and to propose a larger mechanistic framework in terms of multidimensional gradient. He does not openly criticise the idea of machine-likeness at nano-scale. However, he makes a comparison between macroscopic mechanical machines (such as a watch) and macromolecules (such as a ribosome) and he claims, in line with Moore (2012), that "the movements and the interactions of the parts of the watch explain how the watch works. The parts of a

\footnotetext{
${ }^{26}$ By this term Moore means all those pictures that depict the motion of macromolecules as a linear movement produced solely from their component parts.
} 
protein, like a ribosome, do not stand in the same relations as the parts of a mechanical clock" (Skillings 2015, p. 1145). Although this is undoubtedly correct, we find that it may lead to a misleading idea of 'machine' which is based on a (macroscopic) mechanical machine (like a watch). As we have already emphasised in section 2, a machine is a meta-stable structure consisting of interdependent parts which constrain a flow of energy and matter in order to do work and perform a systemic function. Accordingly, a machine is a kind of structure that encompasses different types of macroscopic and microscopic systems and, therefore, cannot be reduced to a (macroscopic) mechanical machine. In other words, both Moore's (2012) and Skillings' (2015) papers correctly criticise a rough analogy between (macroscopic) mechanical machines and MMs. However, these papers give the impression (Moore more explicitly, whereas Skillings implicitly) that it is wrong to consider artificial nano-devices, biomolecular motors and pumps, and ribosomes as machines at all. We argue that a broader, but at the same time more precise, definition of 'machine' does not prevent us to regard this subset of macromolecules as machines.

In addition to Moore's and Skillings' arguments, Nicholson (2013) also maintains that, if biological macromolecules were machines, they should have an organisation created by an intelligent designer, since "confronted with a machine, one is justified in inferring the existence of an external creator responsible for producing it in accordance with a preconceived plan or design" (Nicholson 2013, p. 671). Nicholson's claim can be dismissed by arguing that the existence of an intelligent designer is a necessary condition for achieving functional organisation in man-made machines (and for defining what is a useful task), but neither the existence of functional tasks nor the origin of the order of the (sub)functions involved in such tasks require an intelligent designer in biological systems. These two aspects may be explained by bearing in mind that biological systems are a very special form of self-sustaining organisation, capable of harbouring functional differentiation and undergoing an evolutionary history.

In a recent paper, Nicholson (2018) criticises the analogy between machines and organisms by offering some arguments taken from thermodynamics. Even though the paper focuses on organisms as wholes, it is possible to apply some criticisms of the machine-likeness of living beings to biological macromolecules. Nicholson argues that there are three important differences between machines and biological organisations. First, "organisms have to constantly exchange energy and matter with their surroundings in order to maintain themselves far from thermodynamic equilibrium. Machines, on the other hand, exist in equilibrium or near-equilibrium conditions, and consequently do not have to constantly exchange energy and matter with their surroundings" (Nicholson 2018, p. 144). Second, machines are characterised by static stability (i.e. they do not need an energy input to preserve their structure), whereas biological organisations "exhibit a dynamical stability, which is based on their capacity to actively maintained a low-entropic "steady-state"" (Nicholson 2018, p. 144). And third, the activity of a machine is temporary because of its switching on/off, while "the actively-maintained steady-state of an organism is fixed and irreversible" (Nicholson 2018, p. 144). Despite being correct, these remarks do not preclude the fact that, within a biological system as a whole, there are parts which exhibit a certain degree of stability in near-to-equilibrium conditions (i.e. self-assembling complex structures) and that, in particular, some biological macromolecules -notably biomolecular motors and pumpshave features (i.e. being near thermodynamic equilibrium, exhibiting static stability and temporary activity, etc. ${ }^{27}$ ) that allow them to be talked about in terms of machines.

\footnotetext{
${ }^{27}$ One good example of this is how $\mathrm{F}_{0} \mathrm{~F}_{1}$ ATPase behaves in brown adipose tissue. The presence of an uncoupling protein (UCP) within the inner mitochondrial membrane dissipates the proton gradient
} 
Thus, we believe that, despite all the aforementioned differences, these features (being near thermodynamic equilibrium, exhibiting static stability and temporary activity) allow us to subsume both macroscopic and microscopic man-made machines and a subset of macromolecules into the concept of 'machine'.

Nicholson is right to point out that biomachines exist within and hinge on a dissipative and autonomous organisation. If biological MMs exist, it is because they contribute to creating and maintaining a network of dependencies, namely a true 'closure of (macromolecular) machines', and this global network (i.e. the cell) exists in far-fromequilibrium conditions (Winning and Bechtel 2018). As a result, in spite of being precarious dynamical macromolecular structures, biomachines are relatively stable, since they are produced, regenerated and repaired within a network that they in turn create and support. Furthermore, as we acknowledge in Section 4.2, biomachines also perform their functions in so far as they coordinate their operations with many other biological processes.

\section{Concluding Remarks}

We have argued that, despite important differences derived from the change of scale, large molecular structures (sometimes, in the form of modules (Raanan et al. 2018)) may be either artificially or naturally assembled into an ordered whole, so as to perform a potentially useful activity. At the microscopic scale, the building blocks that need to be assembled to form the global functional device (i.e. 'the machine') are not inert parts, but intrinsically-active entities, which either human engineers or cellular machinery harness so as to achieve a suitable arrangement. In synthetic bio-engineering, different intrinsically-active macromolecular structures are harnessed to (once assembled) produce certain desired patterns of activity. Moreover, many of these patterns of activity are similar to those of biomolecular motors (myosin, kinesin and dynein) and pumps (ATPases), such as myosin, kinesin and dynein. For all these reasons, we conclude that scale-related differences do not justify dismissing the status of these devices as machines, and that both synthetic and some natural molecular devices can rightly be characterised as such since, ultimately, they are functionally-ordered sets of functional parts that, together, constrain a flow of energy so as to produce a new, more complex and integrated function. Moreover, as in macroscopic machines, in both synthetic and biological macromolecular devices, the combination of functional parts to produce new ordered wholes results in an open domain of functions.

However, here is where the specificities of biological macromolecular machines emerge. As we have stressed, whereas synthetic molecular machines exhibit a pattern of activity that is defined by an external intelligent agent, natural ones define their patterns of activity as a result of the organisation of cell's biochemical network. Since biological MMs perform a function by cooperating with many other similar devices within the biochemical network of the cell, they either support the maintenance of the global cellular organisation or, sooner or later, disappear. As has been pointed out by Arnellos and Moreno (2012), the functionality of cellular macromolecules is maintained by a set of mutually-dependent functional structures. Moreover, since biological MMs are highly vulnerable and constantly need to be supplied with energy, they can be maintained only through operations of repair and reproduction (Collier and Hooker

generated by the complexes of the electron transport chain. When the UCP channel is open, no proton flux goes through the $\mathrm{F}_{0} \mathrm{~F}_{1} \mathrm{ATPase}$ and, therefore, there is no production of ATP molecules, but rather heat production. In this case, the structure of the $\mathrm{F}_{0} \mathrm{~F}_{1}$ ATPase biomolecular machine is maintained, even if the machine does not work and performs no function (i.e. the production of ATP molecules). 
1999). The activity generated by macromolecular structures must be harnessed to produce and continuously repair the system in which they are in turn built (Winning and Bechtel 2018). Recently, Bechtel (personal communication) has pointed out that one crucial difference between synthetic and biological molecular machines is that the former display a pattern of activity which becomes functional only through the external action of human beings who put them into a socially-defined system, whereas biological machines, which are intrinsically autopoietic, become functional by virtue of being produced by (and contributing to the maintenance of) a metabolic organisation.

The reason for this co-dependence between natural molecular machines and the cellular metabolic organisation is that, in a natural context, their respective origins can only be explained in terms of co-evolution. On the one hand, the functionality of biological molecular machines evolved because they were incorporated into a selfmaintaining (SM) system; and on the other, the evolution of the overall dynamics of a SM system is intrinsically linked to the increase in structural and functional complexity of its biological molecular machines. Although geological or other types of abiotic processes played a pivotal role, biological molecular devices only began to perform functional activities within SM systems. Moreover, an SM organisation of mutuallydependent constraints ensured the self-maintenance of biological molecular machines.

Biological machines are highly precarious and their maintenance depends on the maintenance of other cellular mechanisms (i.e. the degradation and replacement of proteins). On the one hand, the cell's biochemical network is maintained by the specific contributions of each machine; and on the other, each biological MM is maintained by its participation in a largely distributed, far-from-equilibrium network (the set of processes and machine activities that constitute the cellular metabolism). The core organisation of biological systems (the living cell) is constituted by a host of molecular machines that participate reciprocally in their respective processes of fabrication, maintenance and operation. We will call this organisation a functional integration of macromolecular machines.

The fact that, as pointed out in section 2, artificial and biological machines are embedded, respectively, in a social and in a biological context is at the root of their functional complexity: even though each component part of a machine plays a functional role in constraining a flow of energy and matter, the systemic function (or mechanism) of a machine is something new and not reducible to the singular operations of the parts of the machine. The interesting role of machines is that they allow an increase of the functional complexity of the organisation where they are produced and to which they contribute to maintain. The organized disposition of the components in a meta-stable structure produces a new systemic function that is different from the underlying subfunctional actions of these components.

Mossio and Moreno (2010) and Moreno and Mossio (2015) have developed the idea that the specific causal regime of living systems is a closure of constraints. Ultimately, this is an extremely difficult task, since the coordination of a complex set of constraints requires regulatory control of the biochemical network of the cell which is established by different molecular mechanisms and biological MMs (Bich et al. 2016, Winning and Bechtel 2018). Here we have argued that a machine is a complex, functionally-ordered set of constraints that together act as a whole, generating a new functional activity.

In sum, the appearance of machines was of paramount importance in prebiotic and biological evolution, because it opened up a new domain of functional diversification: new forms of mechanistically-complex functions could be achieved through different combinations of parts. Without the concept of machine, we could not understand how primitive self-sustaining chemical networks progressively achieved higher degrees of 
complexity, generating new domains of integrated functions on the basis of an ordered combination of functional molecular modules.

\section{References}

Ackermann D, Schmidt TL, Hannam JS, Purohit CS, Heckel A, Famulok M (2010) A double-stranded DNA rotaxane. Nat Nanotechnol 5: 436-442

Andersen H (2012) The case for regularity in mechanistic causal explanation. Synth 189: 415-432

Arnellos A. and Moreno A (2012) How functional differentiation originated in prebiotic evolution. Ludus Vitalis 20: 1-23

Astumian RD (2002) Protein conformational fluctuations and free-energy transduction. Appl Phys A 75: 193-206

Astumian RD (2012) Microscopic reversibility as the organizing principle of molecular machines. Nat Nanotechnol 7: 684-688

Astumian RD (2016) Running on information. Nat Nanotechnol 11: 582-583

Astumian RD and Hänggi P (2002) Brownian Motors. Phy Today 55: 33-39

Astumian RD, Mukherjee S, Warshel A (2016) The Physics and Physical Chemistry of Molecular Machines. Chem Phys Chem 17: 1719-1741

Ballardini R, Balzani V, Credi A, Gandolfi MT, Venturi M (2001) Artificial MolecularLevel Machines: Which Energy To Make Them Work?. Acc Chem Res 34: 445455

Balzani V, Credi A, Ferrer B, Silvi S, Venturi M (2005) Artificial Molecular Motors and Machines: Design Principles and Prototype Systems. Top Curr Chem 262: 127

Bechtel W (2017) Explicating Top-Down Causation Using Networks and Dynamics. Philos Sci 84: 253-274

Bechtel W, Richardson RC (1993 (2010)) Discovering Complexity: Decomposition and Localisation as Strategies in Scientific Research. MIT Press, Cambridge (Mass)

Bechtel W, Abrahamsen A (2005) Explanation: a mechanistic alternative. Stud Hist Philos Biol Biomed Sci 36: 421-441

Bechtel W, Abrahamsen A (2013) Thinking Dynamically About Biological Mechanisms: Networks of Coupled Oscillators. Found Sci 18: 707-723

Bich L, Mossio M, Ruiz-Mirazo K, Moreno A (2016) Biological regulation: controlling the system from within. Biol Phil 31: 237-265

Collier J, Hooker CA (1999) Complexly Organised Dynamical Systems. Open Syst Inf Dyn 6: 241-302

Credi A, Silvi S, Venturi M (2014). Light-Operated Machines Based on Threated Molecular Structures. In: Credi A, Silvi S, Venturi M (eds) Molecular Machines and Motors: Recent Advances and Perspectives. Top Curr Chem, vol 354. Springer, Cham, pp. 1-34

Descartes R (1637 (1999)) Discourse on Method and Related Writings. London, Penguin

Descartes R (1664 (1972)) Treatise of Man. Harvard University Press, Cambridge (Mass)

Dicker JJ, Pennock GR, Shigley JE (2003) Theory of Machines and Mechanisms. Oxford University Press, New York 
Feniouk B, Yoshida M (2008) Regulatory Mechanisms of Proton-Translocating of $\mathrm{F}_{0} \mathrm{~F}_{1} \mathrm{ATP}$ Synthase. In: Schäfer $\mathrm{G}$, Penefsky $\mathrm{H}$ (eds) Bioenergetics: Energy Conservation and Conversion. Springer, Berlin Heidelberg, pp 279-308

Feynman R (1963) Lectures on Physics, Volume I, chapter 46. Addison-Wesley, Boston Glennan S (1996) Mechanisms and the Nature of Causation. Erkenntnis 44: 50-71

Illari P (2013) Mechanistic Explanation: Integrating the Ontic and Epistemic. Erkenntnis 78: 237-255

Kaplan DM (2015) Moving parts: the natural alliance between dynamical and mechanistic modeling approaches. Biol Phil 30: 757-786

Kinoshita M (2016) Mechanism of Functional Expression of the Molecular Machines. Springer, Singapore

Lehn JM (1995) Supramolecular Chemistry: Concepts and Perspectives. VCH, New York

Levy A (2014) Machine-Likeness and Explanation by Decomposition. Phil Impr 14: 115

Levy A, Bechtel W (2013) Abstraction and the Organization of Mechanisms. Philos Sci 80: 241-261

Machamer P, Darden L, Craver CF (2000) Thinking about Mechanisms. Philos Sci 67: $1-25$

Moore PB (2012) How Should We Think About the Ribosome?. Ann Rev Biophys 41: $1-19$

Moreno A, Mossio M (2015) Biological Autonomy: A Philosophical and Theoretical Enquiry. Springer, Dordrecht

Mossio M, Saborido C, Moreno Bergareche A (2009) An organizational account of biological functions. Br J Philos Sci 60: 813-841

Mossio M, Moreno A (2010) Organisational Closure in Biological Organisms. Hist Phil Life Sci 32: 269-288

Nicholson DJ (2012) The concept of mechanism in biology. Stud Hist Phil Biol Biomed Sci 43: $152-163$

Nicholson DJ (2013) Organism $\neq$ Machines. Stud Hist Phil Biol Biomed Sci 44: 669678

Nicholson DJ (2018) Reconceptualizing the Organism: From Complex machine to Flowing Stream. In: Nicholson DJ, Dupré J (eds) Everything Flows: Towards a Processual Philosophy of Biology. Oxford University Press, Oxford, pp. 139-166

O’Mahony CT, Farrell RA, Goshal T, Holmes JD, Morris MA (2011) The Thermodynamics of Defect Formation in Self-Assembled Systems. In: MorenoPiraján JC (ed) Thermodynamics - Systems in Equilibrium and Non-Equilibrium (pp. 279-306). InTech, Rijeka, pp. 279-306

Polanyi M (1968) Life's Irreducible Structure. Science 160: 1308-1312

Raanan H, Pike DH, Moore EK, Falkowski PG, and Nanda V (2018) Modular origins of biological electron transport chains. https://doi.org/10.1073/pnas.1714225115

Reuleaux F (1876) Kinematics of Machinery: Outlines of a Theory of Machines. Macmillan, London

Rorick MM, Wagner GP (2011) Protein Structural Modularity and Robustness Are Associated with Evolvability. Genome Biol Evol 3: 456-475

Sauvage JP, Dietrich-Buchecker C (1999) Molecular catenanes, rotaxanes, and knots: a journey through the world of molecular topology. Wiley-VCH, Weinheim

Simón A, Bataller A, Cabrera JA, Ezquerro F, Guerra AJ, Nadal F, Ortiz A (2016) Fundamentals of Machine Theory and Mechanisms. Springer, Cham (ZG) 
Skillings DJ (2015) Mechanistic Explanation of Biological Processes. Philos Sci 82: 1139-1151

Trifonov EN, Frenkel ZM (2009) Evolution of protein modularity. Curr Opin Struct Biol 19: 335-340

Valero J, Lohmann F, Famulok M (2017) Interlocked DNA topologies for nanotechnology. Curr Opin Biotech 48: 159-167

Wilkens S (2000) $\mathrm{F}_{0} \mathrm{~F}_{1}$-ATP Synthase - Stalking Mind and Imagination. J Bioenerg Biomembr 32: 333-339

Wimsatt WC (1986) Forms of Aggregativity. In: Donagan A, Perovich AN, Jr, Wedin MV (eds) Human Nature and Natural Knowledge. Springer, Dordrecht, pp. 259291

Winning J, Bechtel W (2018) Rethinking Causality in Biological and Neural

Mechanisms: Constraints and Control. Minds Mach. https://doi.org/10.1007/s11023$\underline{018-9458-5}$

Zednik C (2011) The Nature of Dynamical Explanation. Philos Sci 78: 238-263 